\title{
Pleomorphic Xanthoastrocytoma, Anaplastic Pleomorphic Xanthoastrocytoma, and Epithelioid Glioblastoma: Case Series With Clinical Characteristics, Molecular Features and Progression Relationship
}

\section{Zhiying Lin}

Southern Medical University Nanfang Hospital

\section{Runwei Yang}

Southern Medical University Nanfang Hospital

\section{Haojie Zheng}

Southern Medical University Nanfang Hospital

\section{Zhiyong Li}

Southern Medical University Nanfang Hospital

\section{Guozhong Yi}

Southern Medical University Nanfang Hospital

Huixia Han

Southern Medical University Nanfang Hospital

Qianchao Wu

Genomicare Biotechnology

\section{Songtao Qi}

Southern Medical University Nanfang Hospital

Yawei Liu

Southern Medical University Nanfang Hospital

Huang Guanglong ( $\nabla$ hgl1020@163.com )

Southern Medical University Nanfang Hospital https://orcid.org/0000-0002-1589-9202

\section{Research}

Keywords: pleomorphic xanthoastrocytoma, anaplastic pleomorphic xanthoastrocytoma, epithelioid glioblastoma, clinical characteristics, molecular features, progression relationship

Posted Date: November 5th, 2020

DOl: https://doi.org/10.21203/rs.3.rs-99690/v1 
License: (c) (i) This work is licensed under a Creative Commons Attribution 4.0 International License. Read Full License 


\section{Abstract}

Background: Pleomorphic xanthoastrocytoma (PXA), anaplastic pleomorphic xanthoastrocytoma (APXA), and epithelioid glioblastoma (E-GBM) show overlapping features. However, little is known about their clinical characteristics, molecular features and progression relationship.

Methods: Fourteen patients diagnosed at Nanfang Hospital from 2016 to 2019 were enrolled, including eleven PXA patients, two A-PXA patients, and one E-GBM patient. All tumor tissue samples of fourteen patients were examined by immunohistochemistry staining (MGMT, VEGF, BRAF-V600E, etc.). The recurred tumor tissue of the E-GBM patient arising from A-PXA was detected for 11 glioma markers (MGMT, BRAF-V600E, etc.) and chromosome 1p/19q by next generation sequencing (NGS).

Results: The mean age of 13 patients with PXA or A-PXA was 25.4 years, twelve of whom were burdened with tumors at supratentorial regions. VEGF showed positive expression in the tumor samples of 13 patients, MGMT positive in 10 patients, and BRAF-V600E positive in 7 patients. As for the tumor sample of the E-GBM patient survived for up to 10 years after the fourth resections, BRAF V600E was wild-type in the sample obtained from the first surgery while it was mutant in the second, third, and fourth surgery. In the contrast, the promoter status of MGMT in four operations were unmethylated. The NGS results showed that the mutation frequencies of BRAF V600E in the second surgery, the third surgery and the fourth surgery were $14.06 \%, 9.13 \%$ and $48.29 \%$ respectively.

Conclusions: Collectively, the results suggest that patients with A-PXA may relapse multiple times and eventually progress to E-GBM with BRAF-V600E mutation.

\section{Introduction}

Pleomorphic xanthoastrocytoma (PXA) is a low-grade brain tumor (WHO II), which was first reported by Kepes et al in 1979 [1]. PXA accounts for less than 1\% of all brain tumors [2], and most of the tumors are located in the supratentorial compartment, mainly in the temporal lobe, followed by the frontal lobe and parietal lobe [3]. On imaging, these tumors showed solid enhanced nodules, often accompanied by eccentric peripheral cystic components and peripheral edema [4]. Histologically, these pleomorphic tumors show different characteristics: spindle cells, multinucleated and fibrous giant cells, eosinophilic granular bodies, polygonal cells and lipid-laden xanthomatous astrocytes [1, 3]. PXA has a favorable prognosis, with a 10-year overall survival rate of approximately $70 \%$ [5-7]. However, $5 \%-20 \%$ of patients with PXA have a recurrence with malignant transformation. PXA rarely transforms into anaplastic pleomorphic xanthoastrocytoma (A-PXA) or glioblastoma (GBM) [8].

A-PXA is similar to PXA in the signs and symptoms but may exhibit aggressive biological behavior with a relatively poorer prognosis than PXA $[9,10]$. The frequency of BRAF V600E mutation is lower compared to PXA and not significantly different between pediatric and adult, but the prognostic significance of the mutation is not clear $[2,11]$. PXA and A-PXA are usually seen in children and young people [1]. No specific epidemiological data are available regarding A-PXA compared with PXA. Although some A-PXA may 
develop from the malignant progression of PXA, the sequence of its potential genetic events has not been determined [12]. The acquisition of the molecular characteristics of PXA and A-PXA helps to reveal the genetic evolution in these tumors.

Epithelioid glioblastoma (E-GBM) is a rare GBM variant newly added to IDH-wild type GBM in 2016 WHO classification [10]. Although the BRAF V600E mutation was rarely found in GBM, it had been identified at a relatively high frequency (50\%) in E-GBM [13]. It is unclear what genetic mechanism may cause the appearance of epithelioid morphology. E-GBM is similar to A-PXA and exhibits epithelioid characteristics. At the histological and molecular level, A-PXA may be difficult to distinguish from E-GBM, because the two tumor types usually show BRAF V600E mutations. E-GBM may coexist with PXA [14] and a case report of E-GBM arising from a PXA has recently been reported, but the relationship between E-GBM and malignant progression in PXA requires further clarification [15]. PXA, A-PXA, and E-GBM show overlapping features, for example, BRAF V600E mutant, IDH1 wild-type, TP53 wild-type, etc [16-22]. In addition, there are overlapping histological features among E-GBM, PXA, and A-PXA, such as discohesive epithelioid patterns of tumor cells, perivascular and intercellular lymphocyte infiltration. Occasionally, it is difficult to distinguish these two tumors [23].

We analyzed the clinical and molecular characteristics of PXA/A-PXA and summarized the possible genetic evolution types of PXA and A-PXA. Then through the study of the characteristics of the E-GBM patient arising from A-PXA after three times recurrences, we speculated the possible malignant progression of A-PXA. Histological and molecular analysis can be performed during the first resection and subsequent recurrences. In histology, the E-GBM patient was diagnosed as A-PXA in the first, second, and third surgical specimens but E-GBM in the fourth surgical specimen. The samples of four operations of this E-GBM was detected by next generation sequencing (NGS).

\section{Materials And Methods}

\section{Case Collection and Histopathology Information}

The Institutional Review Board of Nanfang Hospital affiliated to Southern Medical University approved the study. 11 PXA, 2 A-PXA, and 1 E-GBM diagnosed by pathologists from 2016 to 2019 in Nanfang Hospital were collected. In this study, the clinical data of age, gender, pathological diagnosis (according to the WHO 2016 Central nervous system tumor classification), anatomic location of glioma, tumor size, and pathological molecular markers were collected. Three neuro-oncologist reviews medical records for neuroimaging features, treatment options, and survival data. All specimens' slides were reviewed by pathologists to confirm the diagnosis of all cases. For the diagnosis of PXA, the criteria determined by Brat et al. [3] were used. All our cases satisfied the diagnostic criteria for PXA and A-PXA, respectively: mitotic activity of PXA was $\leq 5$ mitoses per 10 high-power fields (HPF) [12], A-PXA based on either mitotic rate $>5 / 10$ HPFs or presence of necrosis $[2,11]$. GBM displays nuclear atypia, cellular pleomorphism, mitotic activity, a diffuse growth pattern, microvascular proliferation and/or necrosis [10]. Besides, we conducted glial fibrillary acidic protein (GFAP) immunotherapy in all cases. Immunocytochemical 
detection of GFAP can identify the astrological nature of tumor cells and thus support the exclusion of non-glial neoplasms resembling PXA or A-PXA [6].

\section{Molecular Detection}

The pathological molecular markers mainly included GFAP (OriGene, monoclonal, 1:100 dilution), epithelial membrane antigen (EMA, OriGene, monoclonal, 1:100 dilution), 06-methylguanine-DNA methyltransferase (MGMT, OriGene, monoclonal, 1:100 dilution), neuronal nuclei (Neu-N, OriGene, monoclonal, 1:100 dilution), oligodendrocyte transcription factor 2 (Oligo-2, OriGene, monoclonal, 1:100 dilution), epidermal growth factor receptor (EGFR, OriGene, monoclonal, 1:100 dilution), vascular endothelial growth factor (VEGF, OriGene, polyclonal, 1:100 dilution), isocitrate dehydrogenase 1 (IDH1, OriGene, monoclonal, 1:100 dilution), Ki-67 (OriGene, monoclonal, 1:100 dilution), ATRX chromatin remodeler (ATRX, OriGene, polyclonal, 1:100 dilution), CD34 molecule (CD34, OriGene, monoclonal, 1:100 dilution), Synaptophysin (Syn, OriGene, monoclonal, 1:100 dilution), BRAF V600E (Roche, monoclonal, 1:100 dilution), H3K27M (OriGene, polyclonal, 1:100 dilution), Neurofilament (NF, OriGene, monoclonal, 1:100 dilution), Chromogranin A (CgA, OriGene, monoclonal, 1:100 dilution), CD20 (OriGene, monoclonal, 1:100 dilution), Cytokeratin (CK, OriGene, monoclonal, 1:100 dilution), CD30 (OriGene, monoclonal, 1:100 dilution), Vimentin (OriGene, monoclonal, 1:100 dilution), Capicua (CIC, OriGene, monoclonal, 1:400 dilution), far upstream element binding protein 1 (FUBP1, OriGene, monoclonal, 1:400 dilution), Desmin (OriGene, monoclonal, 1:100 dilution) and Reticular Fibres (abcam, monoclonal, 1:500 dilution) .

\section{Fluorescence in situ hybridization (FISH) Detection}

We performed FISH on the formalin-fixed and paraffin-embedded (FFPE) tissue sections using the same commercial 1p/19q probes (Vysis paired probes 1p36/1q25 and 19q13/19p13, Abbott Laboratories). Briefly, $5-\mu \mathrm{m}$-thick formalin-fixed, paraffin-embedded sections were deparaffinized, pre-treated with Vysis Pretreatment Solution (1N, NaSCN, Vysis 32-801200, Abbott Molecular Inc.) and digested in protease solution (Vysis 32-801200, Abbott Molecular Inc.). The probe mix (5 to $15 \mu \mathrm{l}$ ) was added to each slide according to the manufacturer's instructions. Target DNA and probes were codenatured at $74^{\circ} \mathrm{C}$ for 5 minutes and incubated at $37^{\circ} \mathrm{C}$ overnight in a humidified hybridization chamber (ThermoBrite, Abbott Molecular Inc.). Post-hybridization washes were performed in $2 x \mathrm{SSC} / 0.3 \% \mathrm{NP}-40$ for 2 minutes at $75^{\circ} \mathrm{C}$. Finally, the slides were air-dried and counterstained with 4,6-diamidino-2-phenylindole (DAPI, Cell Signaling Technology, \#4083).

\section{Tissue Processing and NGS Panel Genotyping}

For the IDH1, IDH2, ATRX, telomerase reverse transcriptase (TERT), tumor protein p53 (TP53), MGMT, H3.3 histone A (H3F3A), H3 clustered histone 2 (HIST1H3B), B-Raf proto-oncogene (BRAF), EGFR, and phosphatidylinositol-4,5-bisphosphate 3-kinase catalytic subunit alpha (PIK3CA) of the special case of the E-GBM patient, the NGS Panel sequencing and analysis were performed at the Genomics Laboratory of GenomicCare Biotechnology (Shanghai, China). For thawed soft tissue or blood, DNA was extracted using the Maxwell RSC Blood DNA Kit (cat\# AS1400, Promega, Madison, WI, USA) on a Maxwell RSC system (cat\# AS4500, Promega). For the FFPE tissue, DNA was extracted using the MagMAX FFPE 
DNA/RNA Ultra Kit (cat\# A31881, ThermoFisher, Waltham, MA, USA) on a KingFisher Flex system (ThermoFisher). The extracted DNA was sheared using a Covaris L220 sonicator, then the library preparation and capture was done using Tecan EVO 150 (Thermofisher), and sequenced on an Ion S5 sequencer (Thermofisher) to generate paired-end reads. After removing adapters and low-quality reads, the reads were aligned to the National Center for Biotechnology Information (NCBI) human genome reference assembly hg19 using the Burrows-Wheeler Aligner alignment algorithm and further processed using the Genome Analysis Toolkit (GATK, version 3.5), including the GATK Realigner Target Creator to identify regions that needed to be realigned. Somatic single-nucleotide variants (SNV), insertion and deletion (Indel), and copy number variation (CNV) were determined using the MuTect/ANNOVAR/dbNSFP31, VarscanIndel, and CNV nator software respectively as reported in Zang et al. [24].

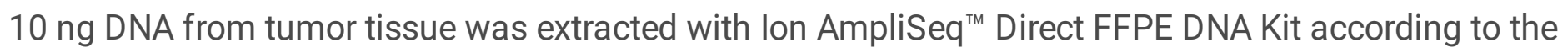

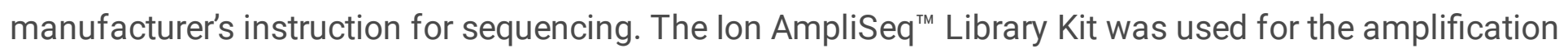
of ten genes (IDH1, IDH2, ATRX, TERT, TP53, H3F3A, HIST1H3B, BRAF, EGFR, and PIK3CA) and the preparation of amplicon libraries. Amplicon libraries were processed using the lon $540^{\mathrm{TM}}$ Kit-Chef and then sequenced by the Thermo Fisher Scientific lon S5 ${ }^{\text {TM }}$ System panel platform. Sequencing reads were mapped to hg19 with BWA using the default parameters. SNV annotation was performed by ANNOVAR and indels detection was analyzed with Varscan 2.4.2. Candidate somatic SNV and indels were identified if they satisfied the following conditions: the sequencing depth was larger than 300 , then they were supported by at least 30 reads; the mutation frequency was larger than $1 \%$.

MGMT promoter methylation status detection was carried out on FFPE tissue by methylation-specific polymerase chain reaction (MS-PCR). $200 \mathrm{ng}$ purified DNA was subjected to bisulfite conversion using the EZ-96 DNA Methylation-Lightning MagPrep kit (cat\# D5046, Zymo Research, Irvine, CA USA) and amplified using EpiMark Hot Start Taq DNA polymerase (cat\#M0490, NEB) in PCR with sense (5'CCATCTCATCCCTGCGTGTCTCCGACTCAG-Index-GATATGTTGGGATAGTT-3') and anti-sense (5'CCTCTCTATGGGCAGTCGGTGAT -CCCAAACACTC

ACCAAAT-3') primer oligos. The sequences matched to the MGMT promoter region were underlined. The PCR products were purified using Agencourt AMPure XP magnetic beads (cat\# A63881, Beckman Coulter, Brea, CA, USA) and subjected to library preparation and sequencing similar to the above in the NGS panel section. Sequenced reads were aligned to NCBI human genome reference assembly hg19 using Bowtie2 (version 2.2.8), Samtools (version 1.3.1), and Bismark version 0.16 .3 to identify methylated cytosine.

Multiplex PCR based on the NGS was used to confirm true chromosome (chr) 1p / 19q status. First of all, 10 single nucleotide polymorphisms (SNPs) are selected on 1p and 10 SNP is located in 19q.13.3 section on chr 19q. There are 5 SNP sections located in 19q.13.3 section on chr 19q. These two regions were used to judge whether loss of heterozygosity occurs in common deletion regions, and combined with other SNP. We can judge whether loss of heterozygosity occurs in the whole long arm or severed arm. The heterozygosity distribution of each SNP in the East Asian population refers to the gnomAD_exome_EAS 
database, and the selection criteria were that the probability that the SNP was heterozygous in the population is close to $50 \%$. The distance of SNP in the genome is more than $300 \mathrm{~kb}$. Then primers and amplification fragments were designed. According to the selected sites, primers suitable for multiple amplification were designed, and the $T$ at the beginning of the second base at the $3^{\prime}$ end of each primer was modified to $U$. The samples were amplified by Platinum Multiplex PCR Master Mix of Thermofisher and treated with uracil DNA glycosylase (UDG) to make the single strand of $U$ form an Apyrimidine (AP) site. Then the library is constructed and sequenced, the lon torrent of Thermofisher is used for sequencing, and the lonXpress series connector of lon torrent is used to construct the sequencing library. Finally, the sequencing results are analyzed, and the sequence data were compared with the hg19 sequence to get the allele frequency (AF) percentage of each SNP. In this study, the homozygous type is defined as $95 \%-100 \%$ or $0-5 \%$, and the heterozygous type is defined as $45 \%-55 \%$. If it is outside the above range, it is considered that loss of heterozygosity $(\mathrm{LOH})$ has occurred in the SNP. If both $1 \mathrm{p}$ and $19 q$ are $\mathrm{LOH}$, it is considered to be chr $1 \mathrm{p} / 19 \mathrm{q} \mathrm{co}-\mathrm{LOH}$.

\section{Statistical analysis}

The SPSS statistical software package (version 25, IBM Corp.) was used for all analyses. Categorical variables are shown as numbers and percentages, while continuous variables are shown as the mean and standard deviation (SD).

\section{Results}

\section{Clinical and histopathologic features of PXA/A-PXA}

A total of 13 patients who were diagnosed with PXA or A-PXA in Nanfang Hospital were utilized to analyze and summarize the clinicopathologic and molecular features. One E-GBM patient arising from APXA was used to analyze the possible disease progression process of A-PXA.

There were five females and eight males diagnosed with PXA/A-PXA. Patients were aged 7 to 53 years, including four pediatric cases. The supratentorial to subtentorial ratio is 12.00 . The mean age of patients was 25.40 years old, and the mean diameter of the tumor was $3.50 \mathrm{~cm}(\mathrm{SD}=0.48 \mathrm{~cm})$.

In our study, it was found that in all patients GFAP and VEGF were positive expressions, chr 1p/19q was intact, and H3K27M was negative expression. 6 of 7 patients were CD34 positive and six patients were S100 positive, 9 of 13 patients showed positive $\mathrm{Ki}-67$ in the range of $0-3 \%, 7$ of 13 patients were BRAF V600E mutant, 5 of 13 patients were EMA negative, 3 of 13 patients were MGMT promoter methylated. Detailed information for this cohort of PXA and A-PXA patients is recorded in Table 1.

\section{E-GBM patient arising from A-PXA}

In order to analyze the possible malignant progression of A-PXA, we selected a patient with E-GBM relapsed from A-PXA to study (Table 2). The E-GBM patient was a 40-year-old woman who visited Nanfang hospital owing to a headache and blurred vision for half a month on November 30th, 2010. 
Magnetic resonance imaging (MRI) showed the left parietal lobe occupied a space that was considered to be tumorous changes with a size of about $5.8 * 3.1 * 4.1 \mathrm{~cm}$ and a clear border. It could be seen that a reinforced nodule with a size of about $2.4 * 0.9 \mathrm{~cm}$ was thickened adjacent to the meninges, and the space-occupying effect was obvious. The left ventricle was compressed and the midline structure was shifted to the opposite side (Fig. 1). After the first surgery, the pathological diagnosis was A-PXA. The first surgical specimen exhibited that the arrangement of cancer cells was compact and flaky, with transparent pronucleus cytoplasm, different sizes, heterogeneity, no obvious necrosis and local vascular endothelial cell proliferation (Table 3). The molecular markers are characterized by Ki-67 (label index: 10\%), GFAP (positive), MGMT (unmethylated), EMA (negative), oligarch - 2 (positive), IDH1 (wild type) and BRAF V600E (wild type) (Fig. 2). The FISH detection showed that chr 1p and 19q were intact.

The patient received postoperative radiotherapy and temozolomide (TMZ) chemotherapy. Follow-up MRI scans were carried out every year. There were not any recurrences of MRI in six years after the first operation. However, six months after the follow-up in June 2016, the patient experienced right limb weakness. The preoperative MRI suggested tumor recurrence on the left parietal lobe (Fig. 1B). After the second surgery, the pathological diagnosis was A-PXA. (Fig. 1). After the second surgery, the pathological diagnosis was A-PXA. The cancer cells are densely packed, with a proliferation of small blood vessels in the interstitium and local collagen fibers. Acute inflammatory cell infiltration and mitosis are seen. Compared with the first postoperative tumor tissue, the second postoperative immunohistochemical results showed that most of the molecular markers are similar, BRAF V600E was mutant, the newly added markers were Neu-N (negative), TP53 (wild-type), VEGF (positive), EGFR (positive), ATRX (wild-type), CD34 (partial positive), CD68 (positive)(Figure S1A), Reticular Fibres (positive), but the label index of Ki-67 fell to $5 \%$ (Fig. 2A). FISH detection of $1 p / 19 q$ was the same as the first postoperative result. The patient received postoperative radiotherapy and temozolomide (TMZ) chemotherapy.

Eight months after the second surgery, the patient experienced weakness of lower extremity for more than one month. The preoperative MRI showed recurrent glioma at the left parietal lobe (Fig. 1). After the third surgery, the pathological diagnosis was A-PXA, WHO III. The size and shape of the cancer cells were different. There was hyperplasia of small blood vessels in the stroma, local hemorrhage, and no obvious necrosis. Compared with the tumor tissue of the second postoperation, the third postoperative immunohistochemical results demonstrated that most markers were similar, except that the Ki-67 index raised to $8 \%$, BRAF V600E was mutant, and Neu-N was changed from negative to residual neurons positive (Fig. 2). FISH detection showed that chr 1p and 19q were intact. One month after the operation, the patient received radiotherapy and TMZ chemotherapy. The dose of the first cycle of TMZ chemotherapy was $200 \mathrm{mg}\left(150 \mathrm{mg} / \mathrm{m}^{2}\right)$ orally once a day for 6 days. In the second to the sixth cycles, the dose was $300 \mathrm{mg}\left(200 \mathrm{mg} / \mathrm{m}^{2}\right)$ orally once a day for 5 days for every cycle.

After two years, the patient experienced weakness in the right limb, which gradually increased for more than a month. Four limbs have involuntary movement, left muscle strength is normal, the muscle strength grade of the right upper limb is zero, lower limb is IV. Preoperative MRI head perfusion enhanced scan indicated left frontal-parietal lobe occupation of $2.0 \mathrm{~cm} \times 1.2 \mathrm{~cm}$, considering the possibility of tumor 
recurrence (Fig. 1). After the fourth surgery, the pathological diagnosis was E-GBM, WHO IV, IDH-1 wildtype. The fourth tissue is densely packed with cancer cells, small blood vessels and palisade necrosis. Compared with the third tumor tissue, the fourth postoperative immunohistochemistry demonstrated that GFAP, MGMT, EMA, IDH1, Oligo-2, EGFR, BRAF V600E, TP53, and ATRX were similar. The newly add markers were CIC (wild-type), FUBP1 (positive), H3K27M (negative expression) (Figure S1B). The Ki-67 index raised to $35 \%$, Neu-N was changed from residual neurons positive to negative, and VEGF changed from positive expression to negative expression (Fig. 2B). FISH detection showed that chr $1 p$ and $19 q$ were intact. Postoperative radiotherapy and TMZ intensive regimen were considered as adjuvant chemotherapy. The dose of the first cycle of TMZ chemotherapy was $200 \mathrm{mg}\left(150 \mathrm{mg} / \mathrm{m}^{2}\right)$ orally once a day for 6 days. In the second to sixth cycles, the dose was $300 \mathrm{mg}\left(200 \mathrm{mg} / \mathrm{m}^{2}\right)$ orally once a day for 5 days for every cycle. Detailed information for the pathology characteristics and molecular features of the E-GBM patient was recorded in Supplementary Table 3 and Supplementary Table 4, respectively.

\section{NGS panel analysis}

We then performed the NGS panel on the E-GBM and A-PXA specimens from this patient. The detected glioma-related markers and chromosomes were IDH1, IDH2, ATRX, TERT, TP53, H3F3A, MGMT, BRAF, EGFR, PIK3CA, HIST1H3B, and chr 1p/19q (Supplementary Table 5). The results indicated that the mutation frequencies of BRAF-V600E in the second specimen, the third specimen, and the fourth specimen were $14.06 \%, 9.13 \%$, and $48.29 \%$ respectively. IDH1, IDH2, ATRX, TERT, TP53, H3F3A, HIST1H3B, MGMT, EGFR, and PIK3CA were wild-type, and the chr 1p/19q was intact.

\section{Discussion}

Our study of PXA and A-PXA is a small part of a glioma that is already rare, which is why the number of cases in our study is limited. In most of our cases, A-PXA was removed for the first time. A-PXA may occur during the initial resection, with anaplastic characteristics, or develop from PXA grade to Amura PXA, but the prognosis of both is usually poor $[22,25,26]$.

In our research on the clinical characteristics, molecular features of PXA and A-PXA, we found that $53.85 \%$ (7/13) were confirmed BRAF V600E mutation, VEGF was a positive expression, H3K27M was a negative expression, and $85.71 \%$ (6/7) patients were CD34 positive expression. Similar to previous studies [27, 28], BRAF V600E mutations did not occur $100 \%$ in PXA, approximately $60 \%$. There was currently no literature about the relationship between PXA/A-PXA and VEGF. In our study, all PXA/A-PXA expressed VEGF, which may be the common genetic evolution of PXA/A-PXA.

We studied the possible malignant progression of A-PXA through a case of E-GBM recurring from A-PXA. We described the case of E-GBM developing at the site of a previously resected A-PXA with the BRAF V600E mutation 10 years after the initial surgery. Histologically, E-GBM was reported to possess reticulin investiture to variable degrees similar to the present case, which may prompt diagnostic consideration of A-PXA. However, E-GBM showed more cytologically uniform cells and a lack of eosinophilic granular bodies [2]. In addition, the clinical course of this case was consistent with that of E-GBM tumor, including 
invasive progression and cranial spinal cord dissemination [16, 22, 25, 26]. A-PXA and E-GBM are similar in histology, immunohistochemistry, molecular and clinical characteristics. Although PXA rarely transforms into A-PXA or GBM [8], there have been some related studies of PXA transformed into GBM so far, for example, 1) a case of secondary disease with an IDH1 mutation had been reported, which is a common type of molecular feature of secondary GBM secondary to astrocyte tumors [2]. 2) Shingo Tanaka et al. described a case of E-GBM developing at the site of a previously resected PXA with the BRAF V600E mutation 13 years after the initial surgery [15]. However, to our knowledge, this is the first report on E-GBM caused by A-PXA.

For this case of E-GBM, histological studies and molecular analyses were performed at the primary resection and three relapses (Fig. 3). Through immunohistochemical analysis, it was found that IDH1 was wild-type, MGMT promoter was unmethylated, the BRAF V600E were wild-type (first tissue), mutation (second, third resection, and fourth tissues), and VEGF was positive (second and third tissues), negative (fourth tissue). In order to identify the genetic evolution of E-GBM, we specifically performed the NGS panel tests on tumor tissues in the four resection. According to the results of the NGS panel, the E-GBM patient detected a total of 11 glioma markers and chr 1p/19q, the results indicated that only BRAF V600E was mutant in the second surgery, the third surgery, and the fourth surgery.

IDH1 and TP53 mutations are usually not detected in A-PXA and E-GBM [16-22]. Comprehensive immunohistochemistry and NGS panel results showed that our case did not contain mutations in these genes in A-PXA and E-GBM, indicating that these genes did not affect the progression from A-PXA to EGBM. In the case reported by Shingo Tanaka et al. [15], the MGMT promoter was positive in E-GBM but not PXA. It was speculated that during the transformation from PXA to E-GBM, the state of the MGMT promoter changed from methylated to unmethylated. In contrast, in our case, MGMT promoters in A-PXA and E-GBM were unmethylated, and only $23.08 \%$ of the 13 cases of PXA/A-PXA were MGMT promoter methylated. In this research, we found that the VEGF in the E-GBM patient was positive (second and third resection), negative (fourth resection), and the VEGF of 13 cases of PXA/A-PXA in this study were positive. It may be possible to speculate that the status of VEGF changed from positive expression to negative expression accompanied by the transformation from A-PXA to E-GBM. Further analysis is needed to study whether the genomic level show change during the transformation from A-PXA to E-GBM.

Collectively, this study investigated the possible common clinical pathology and molecular characteristics of PXA and A-PXA. Through a case study, it is suggested that E-GBM may be the outcome of the malignant transformation of A-PXA. As far as we know, this is the first report of E-GBM arising from APXA. In order to clarify the relationship between A-PXA and E-GBM, it is necessary to study more cases.

\section{Abbreviations}

PXA (Pleomorphic xanthoastrocytoma); A-PXA (anaplastic pleomorphic xanthoastrocytoma); E-GBM (Epithelioid glioblastoma ); GBM (glioblastoma); WES (whole exome sequencing); WHO (World Health Organization); NGS (next generation sequencing); GBM (glioblastoma); CNS (Central Nervous System); 
NCBI (National Center for Biotechnology Information); SNV (Somatic single-nucleotide variants); CNV (copy number variation); IDH1 (isocitrate dehydrogenase 1); IDH-wt (IDH-widetype); GFAP (glial fibrillary acidic protein); FFPE (formalin-fixed and paraffin-embedded); EMA (epithelial membrane antigen); MGMT (O6-methylguanine-DNA methyltransferase); Neu-N (neuronal nuclei); Oligo-2(oligodendrocyte transcription factor 2); EGFR (epidermal growth factor receptor); VEGF (vascular endothelial growth factor); CIC (Capicua); FUBP1 (far upstream element binding protein 1).

\section{Declarations}

\section{Acknowledgements}

A particular acknowledgment to the medical staff involved in the study.

\section{Funding}

This study was supported by the Guangdong Science and Technology Department (2016A040403053).

\section{Availability of data and material}

The data analyzed in this study can be accessed by sending a request to the corresponding author.

Author information

\section{Affiliations}

Department of Neurosurgery, Nanfang Hospital, Southern Medical University, Guangzhou 510515, Guangdong, China

Zhiying Lin; Runwei Yang; Haojie Zheng; Zhiyong Li; Guozhong Yi; Songtao Qi; Yawei Liu; Guanglong Huang

The Laboratory for Precision Neurosurgery, Nanfang Hospital, Southern Medical University, Guangzhou 510515, Guangdong, China

Zhiying Lin; Runwei Yang; Haojie Zheng; Zhiyong Li; Guozhong Yi; Songtao Qi; Yawei Liu; Guanglong Huang

\section{Nanfang Glioma Center, Guangzhou 510515, Guangdong, China}

Guozhong Yi; Zhiyong Li; Xian Zhang; Songtao Qi; Guanglong Huang

Department of Pathology, Nanfang Hospital, Southern Medical University, Guangzhou 510515, Guangdong, China

Huixia Han 
Genomicare Biotechnology (Shanghai) Co. Ltd., Shanghai, China

Qianchao Wu

\section{Contributions}

Conception and design of the work: GLH and YWL. Acquisition, analysis and interpretation of data: ZYL, RWY, HJZ, ZYL, GZY, HXH, QCW, STQ, and GLH. Drafting of the manuscript: ZYL, RWY and GLH. Critical revision for important intellectual content: ZYL, RWY, GZY, ZYL, YWL, STQ, and GLH. All authors approved the final version of this manuscript.

\section{Corresponding authors}

Correspondence to Yawei Liu and Guanglong Huang,

\section{Ethics declarations}

\section{Ethics approval and consent to participate}

A written consent was obtained from a patient or legal guardian on behalf of the participants under the age of 16. The Institutional Review Board of Nanfang Hospital affiliated to Southern Medical University approved the study.

\section{Consent for publication}

Written informed consent was obtained from the patients for publication of this case reports. All the authors listed have approved the enclosed manuscript.

\section{Competing interests}

The authors declare that they have no competing interests.

\section{References}

1. Kepes JJ, Rubinstein LJ, Eng LF: Pleomorphic xanthoastrocytoma: a distinctive meningocerebral glioma of young subjects with relatively favorable prognosis. A study of 12 cases.Cancer 1979, 44:1839-1852.

2. Ida CM, Rodriguez FJ, Burger PC, Caron AA, Jenkins SM, Spears GM, Aranguren DL, Lachance DH, Giannini C: Pleomorphic Xanthoastrocytoma: Natural History and Long-Term Follow-Up.Brain Pathol 2015, 25:575-586.

3. Giannini C, Scheithauer BW, Burger PC, Brat DJ, Wollan PC, Lach B, O'Neill BP: Pleomorphic xanthoastrocytoma: what do we really know about it? Cancer 1999, 85:2033-2045.

4. Rippe DJ, Boyko OB, Radi M, Worth R, Fuller GN: MRI of temporal lobe pleomorphic xanthoastrocytoma.J Comput Assist Tomogr 1992, 16:856-859. 
5. Gallo P, Cecchi PC, Locatelli F, Rizzo P, Ghimenton C, Gerosa M, Pinna G: Pleomorphic xanthoastrocytoma: long-term results of surgical treatment and analysis of prognostic factors. $\mathrm{Br} J$ Neurosurg 2013, 27:759-764.

6. Gelpi E, Popovic M, Preusser M, Budka H, Hainfellner J: Pleomorphic xanthoastrocytoma with anaplastic features presenting without GFAP immunoreactivity: implications for differential diagnosis.Neuropathology 2005, 25:241-246.

7. Gil-Gouveia R, Cristino N, Farias JP, Trindade A, Ruivo NS, Pimentel J: Pleomorphic xanthoastrocytoma of the cerebellum: illustrated review.Acta Neurochir (Wien) 2004, 146:1241-1244.

8. Watanabe N, Ishikawa E, Kohzuki H, Sakamoto N, Zaboronok A, Matsuda M, Shibuya M, Matsumura A: Malignant transformation of pleomorphic xanthoastrocytoma and differential diagnosis: case report.BMC Neurol 2020, 20:21.

9. Fukushima H, Nakano Y, Ishii N, Nozuchi N, Okuno T, Yamasaki K, Okada K, Fujisaki H, Kunihiro N, Matsusaka $Y$, et al: Histological and genetic analysis of anaplastic pleomorphic xanthoastrocytoma suspected of malignant progression over a 12-year clinical course.Pathol Int 2019, 69:608-613.

10. Louis DN, Perry A, Reifenberger G, von Deimling A, Figarella-Branger D, Cavenee WK, Ohgaki H, Wiestler OD, Kleihues P, Ellison DW: The 2016 World Health Organization Classification of Tumors of the Central Nervous System: a summary.Acta Neuropathol 2016, 131:803-820.

11. Schmidt Y, Kleinschmidt-DeMasters BK, Aisner DL, Lillehei KO, Damek D: Anaplastic PXA in adults: case series with clinicopathologic and molecular features.J Neurooncol 2013, 111:59-69.

12. Villa C, Miquel C, Mosses D, Bernier M, Di Stefano AL: The 2016 World Health Organization classification of tumours of the central nervous system.Presse Med 2018, 47:e187-e200.

13. Kleinschmidt-DeMasters BK, Aisner DL, Birks DK, Foreman NK: Epithelioid GBMs show a high percentage of BRAF V600E mutation.Am J Surg Pathol 2013, 37:685-698.

14. Alexandrescu S, Korshunov A, Lai SH, Dabiri S, Patil S, Li R, Shih CS, Bonnin JM, Baker JA, Du E, et al: Epithelioid Glioblastomas and Anaplastic Epithelioid Pleomorphic Xanthoastrocytomas-Same Entity or First Cousins? Brain Pathol 2016, 26:215-223.

15. Tanaka S, Nakada M, Nobusawa S, Suzuki SO, Sabit H, Miyashita K, Hayashi Y: Epithelioid glioblastoma arising from pleomorphic xanthoastrocytoma with the BRAF V600E mutation.Brain Tumor Pathol 2014, 31:172-176.

16. Broniscer A, Tatevossian RG, Sabin ND, Klimo P, Jr., Dalton J, Lee R, Gajjar A, Ellison DW: Clinical, radiological, histological and molecular characteristics of paediatric epithelioid glioblastoma.Neuropathol Appl Neurobiol 2014, 40:327-336.

17. Luo W, Li C, Huang J, Chen X, Ma Y, Zeng L: Clinicopathologic changes and molecular finding of epithelioid pleomorphic xanthoastrocytoma: a case report.Int J Clin Exp Pathol 2018, 11:5144-5148.

18. Sugita Y, Shigemori M, Okamoto K, Morimatsu M, Arakawa M, Nakayama K: Clinicopathological study of pleomorphic xanthoastrocytoma: correlation between histological features and prognosis. Pathol Int 2000, 50:703-708. 
19. Martinez R, Carmona FJ, Vizoso M, Rohde V, Kirsch M, Schackert G, Ropero S, Paulus W, Barrantes A, Gomez A, Esteller M: DNA methylation alterations in grade II- and anaplastic pleomorphic xanthoastrocytoma.BMC Cancer 2014, 14:213.

20. Marton E, Feletti A, Orvieto E, Longatti P: Malignant progression in pleomorphic xanthoastrocytoma: personal experience and review of the literature.J Neurol Sci 2007, 252:144-153.

21. Hirose T, Ishizawa K, Sugiyama K, Kageji T, Ueki K, Kannuki S: Pleomorphic xanthoastrocytoma: a comparative pathological study between conventional and anaplastic types. Histopathology 2008, 52:183-193.

22. Okazaki T, Kageji T, Matsuzaki K, Horiguchi H, Hirose T, Watanabe H, Ohnishi T, Nagahiro S: Primary anaplastic pleomorphic xanthoastrocytoma with widespread neuroaxis dissemination at diagnosisa pediatric case report and review of the literature.J Neurooncol 2009, 94:431-437.

23. Furuta T, Miyoshi H, Komaki S, Arakawa F, Morioka M, Ohshima K, Nakada M, Sugita Y: Clinicopathological and genetic association between epithelioid glioblastoma and pleomorphic xanthoastrocytoma.Neuropathology 2018, 38:218-227.

24. Zang YS, Dai C, Xu X, Cai X, Wang G, Wei J, Wu A, Sun W, Jiao S, Xu Q: Comprehensive analysis of potential immunotherapy genomic biomarkers in 1000 Chinese patients with cancer.Cancer Med 2019, 8:4699-4708.

25. Tekkok IH, Sav A: Anaplastic pleomorphic xanthoastrocytomas. Review of the literature with reference to malignancy potential.Pediatr Neurosurg 2004, 40:171-181.

26. Vu TM, Liubinas SV, Gonzales M, Drummond KJ: Malignant potential of pleomorphic xanthoastrocytoma.J Clin Neurosci 2012, 19:12-20.

27. Dougherty MJ, Santi M, Brose MS, Ma C, Resnick AC, Sievert AJ, Storm PB, Biegel JA: Activating mutations in BRAF characterize a spectrum of pediatric low-grade gliomas.Neuro Oncol 2010, 12:621-630.

28. Dias-Santagata D, Lam Q, Vernovsky K, Vena N, Lennerz JK, Borger DR, Batchelor TT, Ligon KL, lafrate AJ, Ligon AH, et al: BRAF V600E mutations are common in pleomorphic xanthoastrocytoma: diagnostic and therapeutic implications.PLoS One 2011, 6:e17948.

\section{Tables}

Due to technical limitations, table 1,2 is only available as a download in the Supplemental Files section.

\section{Figures}




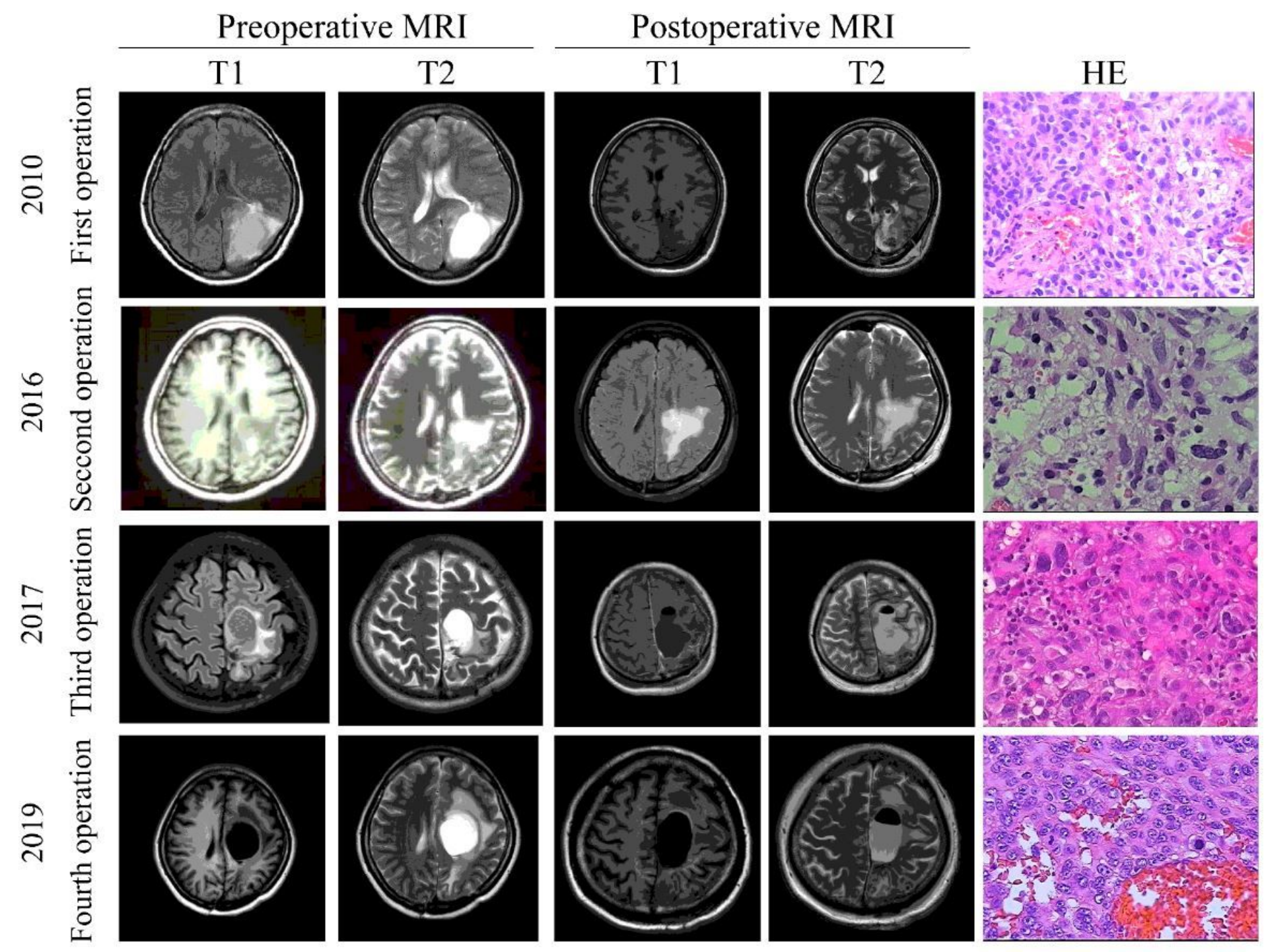

Figure 1

Magnetic resonance imaging (MRI) and hematoxylin-eosin-stained (H\&E) of the four operatives.

Preoperative MRI on the left and postoperative MRI on the right. 


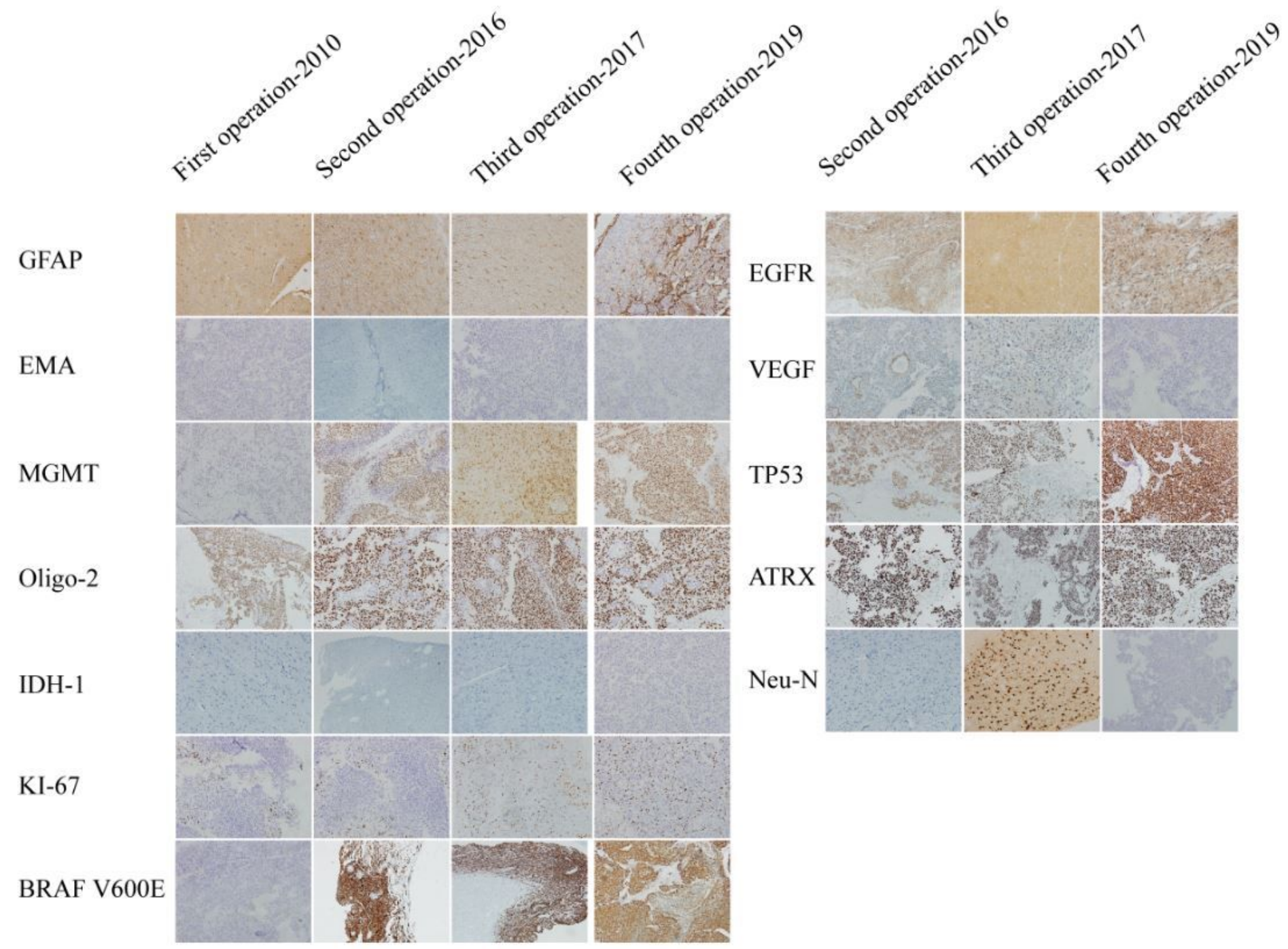

Figure 2

The tumor tissues immunohistochemical features of four postoperations $(A)$ and last three postoperations (B) of A-PXA (the tumor tissues of 2010, 2016, 2017 resection) and E-GBM (the tumor tissues of 2019 resection) areas in a single case. 


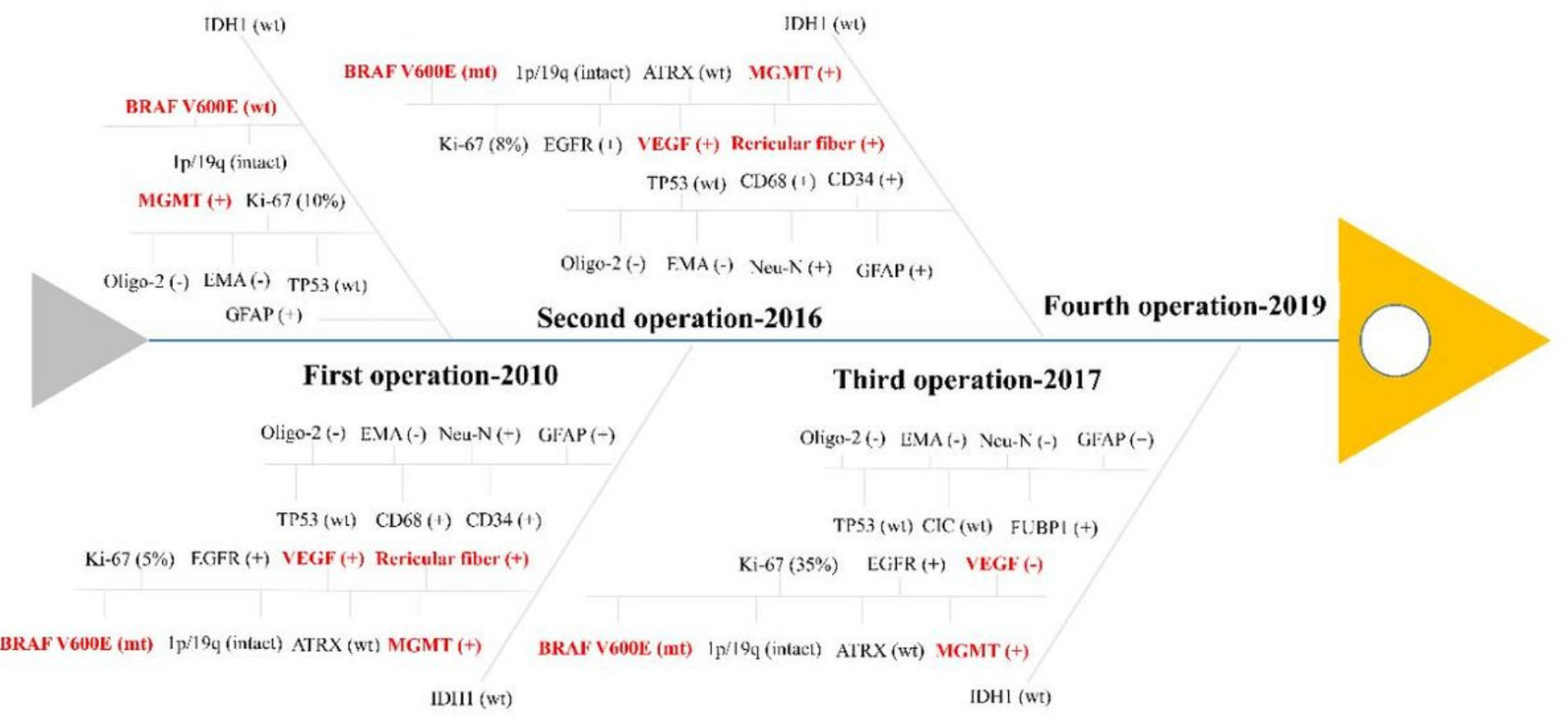

Figure 3

The fishbone diagram of molecular characteristics of the E-GBM for four postoperative tumors.

\section{Supplementary Files}

This is a list of supplementary files associated with this preprint. Click to download.

- FigureS1.tif

- SupplementaryTable1.xlsx

- SupplementaryTable2.xlsx

- SupplementaryTable3.xIsx

- SupplementaryTable4.xlsx

- TABLE1.xIsx

- TABLE2.xIsx 